\title{
Influence of Cathode Diameter on the Operation of a Planar Diode with an Explosive Emission Cathode
}

\author{
Yulia I. Isakova, Galina E. Kholodnaya, \\ and Alexander I. Pushkarev
}

High Technology Physics Institute, Tomsk Polytechnic University, 2 a Lenin Avenue, Tomsk 634028, Russia

Correspondence should be addressed to Alexander I. Pushkarev, aipush@mail.ru

Received 21 July 2011; Revised 20 August 2011; Accepted 24 August 2011

Academic Editor: Pietro Musumeci

Copyright (c) 2011 Yulia I. Isakova et al. This is an open access article distributed under the Creative Commons Attribution License, which permits unrestricted use, distribution, and reproduction in any medium, provided the original work is properly cited.

This paper presents the results of experimental investigations into the current-voltage characteristics of a planar diode with an explosive emission cathode made from graphite. Studies were performed using a TEU-500 pulsed electron accelerator $(350-500 \mathrm{keV}, 100 \mathrm{~ns}, 250 \mathrm{~J}$ per pulse). Duration of diode operation, in a mode when electron current is limited by the emissive ability of the graphite cathode, is $15-20$ ns. The contribution of the cathode periphery to total electron current appears only as an increase in the emissive surface area due to an expansion of explosive plasma. Investigations of an ion diode with a graphite cathode (plane and focusing geometry) were also carried out. Experiments were performed using a TEMP-4M ion accelerator, which forms two nanosecond pulses: the first negative pulse $(150-200 \mathrm{kV}, 300-600 \mathrm{~ns})$ followed by the second positive $(250-300 \mathrm{kV}, 150 \mathrm{~ns})$. Total diode current in the first pulse is well described by the ChildLangmuir law for electron current at a constant rate of plasma expansion, equal to $1.3 \mathrm{~cm} / \mu \mathrm{s}$. It is shown that for an area of flat cathode over $25 \mathrm{~cm}^{2}$, the influence of edge contribution does not exceed measurement error of total diode electron current $(10 \%)$.

\section{Introduction}

Investigations into the behaviour of diodes with an explosive emission cathode during the generation of a pulsed electron beam of current density exceeding $20 \mathrm{~A} / \mathrm{cm}^{2}$ have been carried out since the 1970s. It has been shown that the electron current is limited either by the emission ability of the cathode or by the space charge in the anode-cathode gap (A-K gap) $[1,2]$. Whenever micro explosions occur, a dense plasma forms on the cathode, containing one, two, three or more charged ions and having a temperature of $6-7 \mathrm{eV}$ and a nonuniform space distribution. Directly in the cathode region, concentration of plasma can reach $10^{14} \mathrm{~cm}^{-3}$ or greater, beyond this decreasing inversely with the square of the distance $[3,4]$. From the 
moment voltage is applied until a dense plasma forms on the cathode surface, the electron current is limited by the emissive ability of the cathode. Later, the electron current of the diode is limited only by the space charge of electrons in the A-K gap. It is obvious that the mode of current limitation by space charge is preferable from the standpoint of diode stability. In this mode, the current does not depend on the emissive ability of the cathode but is rather determined only by the geometry of the diode and accelerating voltage. Current distribution over the beam cross-section is also more homogeneous.

In the space charge limitation mode electron current density is defined by the ChildLangmuir law [5]. For a diode with an explosive emission cathode it is necessary to consider a reduction of the A-K gap during the expansion of the plasma surface:

$$
J_{e}(t)=\frac{4 \alpha \varepsilon_{0} \sqrt{2 e}}{9 \sqrt{m}} \cdot \frac{U^{3 / 2}}{d(t)^{2}}=2.33 \cdot 10^{-6} \frac{\alpha U^{3 / 2}}{\left(d_{0}-v t\right)^{2}}
$$

where $J_{e}(t)$ is the electron current density, $U$ is the accelerating voltage, $d_{0}$ is the initial anodecathode gap spacing, $\varepsilon_{0}$ is the absolute permittivity, $m$ is the electron mass, $e$ is the electron charge, $\alpha$ is a current density amplification coefficient $v$ is plasma expansion speed, and $t$-is the current time of voltage.

If in the A-K gap there is a counter ion flow coming from the anode (bipolar flow), a space charge of electrons is partially compensated and the amplification coefficient of current density is $\alpha=1.86$. The perveance of a planar diode with a cathode of radius $r$ is equal to

$$
P v(t)=\frac{J_{e} \cdot S}{U^{3 / 2}}=\frac{2.33 \cdot 10^{-6} \alpha \pi r^{2}}{(d-v t)^{2}}
$$

This ratio is obtained by one-dimensional solution of the Poisson equation without considering the influence of edge contribution. Published experimental data on the contribution of the cathode perimeter to the total electron current of a diode with an explosive emission cathode are contradictory. It is shown in [6] that the voltage-current characteristic for a system with planar electrodes and a single cathode flame that occurs on-site of an artificial microasperity $\left(U_{\mathrm{o}}=20-40 \mathrm{kV}, d=0.3-1 \mathrm{~mm}\right)$ is described as

$$
I=44.6 \cdot 10^{-6} \cdot U^{3 / 2} \cdot\left(\frac{v \cdot t}{d}\right)^{2}=6 \cdot 2.23 \cdot 10^{-6} \cdot U^{3 / 2} \cdot \frac{S}{d^{2}} .
$$

This ratio is obtained from (1.1) provided that the cathode area $S$ is $\pi \cdot(v \cdot t)^{2}$ and the diode current exceeds by 6 times the Childe-Langmuir limit.

Parker et al. [7] have presented the results of a study on a planar diode with a flat graphite cathode during the formation of an electron beam. It was found that the total perveance of the diode was equal to the sum of the perveance of a flat part of the cathode and the perveance of a peripheral region. The perveance of a flat part of the cathode is described by (1.2), in which $\alpha=1$. The electron current from the peripheral region of the cathode is $30-40 \%$ of the total electron current. However, the authors also demonstrated that electron current density was generally uniform over the cross-section, with an increase at the edges of the diode not exceeding 3-5\%. These observations were made when the impedance of the planar diode was increased to $11 \mathrm{Ohms}$ or more while output resistance of the generator of 
nanosecond pulses was $4.7 \mathrm{Ohms}$, that is, in a mode of imbalance between generator and load.

The results of mathematical modelling and experimental investigation of a planar diode with an explosive emission cathode during the formation of a discrete emission surface have been reported by a number of authors $[8,9]$. All studies show a satisfactory agreement between experimental and calculated (at a constant rate of graphite plasma expansion equal to $2 \mathrm{~cm} / \mu \mathrm{s}$ ) values of an average current density for $100 \mathrm{~ns}$. Calculations were performed without taking the edge contribution into account.

The perveance of a planar electron diode with a flat graphite cathode with a diameter of $6.7 \mathrm{~cm}$ was investigated by Roy et al. [10], with studies performed on the KALI-5000 accelerator $(280 \mathrm{kV}, 18 \mathrm{kA}$, duration of $100 \mathrm{~ns})$. As in [7], total diode perveance was equal to the sum of the perveance of the flat part of the cathode and the perveance of the peripheral region. The calculation of the perveance of the flat part of the diode was made using ratio (1.2) for a bipolar flow $(\alpha=1.86)$ with a total expansion rate of both anode and cathode plasma (gap closure rate) equal to $15-19 \mathrm{~cm} / \mu \mathrm{s}$. The electron current from the peripheral region exceeded $40-50 \%$ of total current although the authors note a high uniformity of current density over the cross-section and a lack of current density amplification on the perimeter of the diode. In [10] the calculation of perveance was performed according to the indications of a B-dot probe. However, it can also record current loss caused by the current leaking on the insulator surface and parasitic discharges in the vacuum volume of the diode chamber. In addition, the authors also noticed the presence of a bipolar prepulse with an amplitude of $\pm 90 \mathrm{kV}$, which can cause the formation of plasma and provide a significant reduction in the A-K gap before the arrival of the main voltage pulse.

Roy et al. [11] have considered the processes of electron current generation in a diode with an explosive emission cathode. Typical electron beam parameters were used, being $180 \mathrm{kV}, 26 \mathrm{kA}$, and $100 \mathrm{~ns}$. To eliminate the parasitic influence of a prepulse, an extra spark gap was inserted into the diode connection that separates the cathode and the generator of nanosecond pulses. The total perveance of the diode was then calculated as the sum of the perveance of the flat part of the cathode and that of the peripheral region. The authors noted that a satisfactory agreement between calculated and experimental perveance values was observed only at the end of the pulse.

The perveance of a planar diode with a multipoint explosive emission cathode has been studied by Li et al. [12]. A high-voltage generator of nanosecond pulses $(500 \mathrm{kV}, 50 \mathrm{Ohm}$, $400 \mathrm{~ns}$ ) with an inductive storage was used. Results demonstrated that, if the A-K gap was changed from 17 to $24 \mathrm{~mm}$, the diode perveance during the initial $200 \mathrm{~ns}$ could be described satisfactorily by ratio (1.2) for the unipolar charge carriers flow in the A-K gap $(v=1 \mathrm{~cm} / \mu \mathrm{s}$, $\alpha=1$ ). The authors note that the contribution of the periphery of the planar diode to total electron current appeared only $200 \mathrm{~ns}$ after the beginning of the voltage pulse and did not exceed $5-10 \%$. This study involved changing diode impedance in the initial $300 \mathrm{~ns}$ from 50 up to $60 \mathrm{Ohms}$, that is, in the balance mode of a generator of nanosecond pulses and a diode.

In a later study, Roy et al. [13] carried out an investigation of the perveance of a planar diode with an explosive emission cathode using the KALI-1000 accelerator. The output impedance was $15 \mathrm{Ohms}$, voltage pulse amplitude $300 \mathrm{kV}$, and pulse duration $100 \mathrm{~ns}$. It was found experimentally that the perveance of the diode did not exceed $40-50 \%$ of that calculated from (1.2), with $\alpha=1$.

Hegeler et al. [14] studied the generation of pulsed electron beam on an "Electra" electron beam pumped $\mathrm{KrF}$ laser. Typical operating parameters were $400-550 \mathrm{kV}, 70-110 \mathrm{kA}$, and pulse duration at the base of $180 \mathrm{~ns}$. Two cathode sizes $\left(27 \times 97 \mathrm{~cm}^{2}\right.$ and $\left.35 \times 106 \mathrm{~cm}^{2}\right)$ 
and various cathode materials have been tested. To minimize pinching, the e-beam is guided through the A-K gap by an axial magnetic field of $1.4 \mathrm{kG}$. To measure the electron beam density distribution over the cross-section 8 small-sized Faraday cups (the time resolved measurements) and a radiochromic film (the time-integrated spatial measurements) were used. Studies have shown a high uniformity of current density over the beam section. Electron current density was described by (1.1), in which $\alpha=1$. An increase in current density in 1.5-2 times around the perimeter (beam halo) was noted. Width of the beam halo is on the order of the width of a single section of the Faraday cup array, which is $5 \mathrm{~mm}$. The contribution of the periphery of the cathode to the total electron current did not exceed $8-10 \%$.

The edge contribution should be manifested most fully in a cylindrical diode (pipe in pipe) with an explosive emission cathode. The perveance of a cylindrical diode with a graphite explosive emission cathode of diameter $11 \mathrm{~cm}$ and width $2 \mathrm{~cm}$ has been previously researched in [15]. These studies were performed on the KALI-5000 accelerator set in doublepulse mode, with an initial positive pulse $(142 \mathrm{kV}, 7.3 \mathrm{kA})$ and subsequent negative pulse $(143 \mathrm{kV}, 11.2 \mathrm{kA})$. The authors note a good agreement between experimental and calculated values of perveance during electron beam generation on the second pulse. Calculations were performed using the Langmuir-Blodgett ratio for electron current density between infinitely long cylinders with current limited by space charge. The contribution of edges on the generation of the electron beam was negligible. However, the ratio of the cathode's perimeter length to surface area was $3.1 \mathrm{~cm}^{-1}$, while, for the planar diode of diameter $6.7 \mathrm{~cm}$, whose perveance was studied in [10], the ratio of circumference to area was 5 times less. The contribution of edge on the operation of a cylindrical diode should therefore be much greater, but this is not confirmed experimentally.

In our previous study [16], we reported on the operation of a planar diode with a graphite explosive emission cathode. A change in perveance at the beginning of the pulse here was satisfactorily described by ratio (1.2), provided that current consumption is limited by space charge in the A-K gap. However, the velocity of perveance increase during the pulse considerably exceeded the expected value calculated from the reduction of the A-K gap caused by expanding cathode plasma. These studies were performed using large A-K gaps (15-22 mm), which correspond with the mismatch of output impedance of the generator with the load.

The purpose of this study is to determine the minimum area of a planar diode with a graphite explosive emission cathode in which the contribution of the cathode periphery to the total diode current is negligible.

\section{Experimental Setup}

Experiments were performed on a pulsed electron accelerator, the TEU-500 [17], using the following parameters: accelerating voltage $350-450 \mathrm{kV}$, duration $100 \mathrm{~ns}$, and total kinetic energy of electrons in a pulse up to $250 \mathrm{~J}$. The pulse repetition frequency in the experiments was $0.5-1 \mathrm{~Hz}$. A distinctive feature of the accelerator is the use of a stepup transformer, which connects a double forming line (DFL) and the diode. Saturation of the transformer core during a charge of the DFL makes it possible to significantly reduce the amplitude of the prepulse and thus also its impact on the operation of the diode, without the use of a peaking spark gap. Research has shown that, after predegaussing of the matching transformer core, the shape of the voltage pulse formed by the generator of nanosecond pulses (which includes DFL and the transformer) is close to optimal, taking into account the decrease in diode 
impedance caused by explosive emission plasma expansion [18]. This enables the alignment of the diode with the generator during electron beam generation. Figure 1 shows the diode connection of the accelerator and the location of diagnostic equipment.

To measure the current flowing in the load of the generator of nanosecond pulses, a Rogowski coil with a reverse loop was used, while, to measure voltage, a capacitive divider was used, located in an oil-filled chamber, together with a differential voltage divider [19]. Total electron beam current was measured using a Faraday cup, which was evacuated together with the diode chamber to a pressure of 0.05-0.1 Pa. Measurement of diode currentvoltage characteristics during the generation of electron current pulses of gigawatt power requires an accurate adjustment and calibration of diagnostic equipment. Figure 2 shows the calculated values of diode impedance when the TEU-500 accelerator operates with a resistive load. Calibration was performed upon the generation of a positive polarity voltage pulse, with a parasitic load inductance of $250 \mathrm{nH}$.

Calibration of diagnostic equipment showed a correct reflection of accelerator operation in short-circuit mode $(U=50-60 \mathrm{kV})$, when operating with a resistive load of up to $60 \mathrm{Ohms}(200-250 \mathrm{kV})$ and with the planar diode $(350-500 \mathrm{kV})$. High accuracy of voltage and current measurements and the frequency characteristics of the diagnostic equipment allow the calculation of impedance and perveance of the diode with an accuracy of less than $10 \%$. The measurement error is determined by a statistical processing of experimental data in the Origin program. For the values of the impedance calculated from the experimental waveforms of current and voltage (calibration on an active load), we determined a measurement average and standard deviation.

Investigations were performed using the following planar diode configuration: flat cathodes with diameters of $45 \mathrm{~mm}$ and $60 \mathrm{~mm}$ and as the anode the flat plate collector of a Faraday cup with a diameter of $92 \mathrm{~mm}$ (or a metal grid) was used. The calculation of experimental values of diode perveance can be made from the equation

$$
P v_{\text {calc }}=\frac{I_{e}}{(U-L(d I / d t))^{3 / 2}}
$$

The value of the inductance of cathode holder $L$ was assumed to be equal to $160 \mathrm{nH}$, this value being obtained when calibrating the diode in short-circuit mode.

\section{Investigation of a Diode with a Cathode Diameter of $45 \mathrm{~mm}$}

It has been established that explosive emission plasma at the surface of a cathode extends across the gap (radially to the cathode), leading to an increase in the area of the emission surface. This should also be considered in planar diodes of small area. In this case, the perveance of the diode will be as follows:

$$
P v(t)=\frac{2.33 \cdot 10^{-6} \pi(r+v t)^{2}}{(d-v t)^{2}}
$$

Figure 3 shows the calculated diode perveance with and without consideration of an increase in the cathode emissive area (cathode diameter $45 \mathrm{~mm}$ ). 


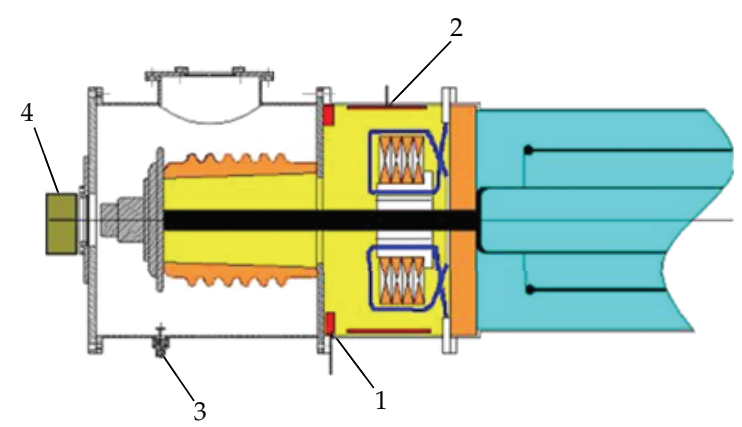

Figure 1: Diagnostic equipment of the pulsed electron accelerator: (1) Rogowski coil, (2) capacitive voltage divider, (3) differential voltage divider, and (4) Faraday cup.

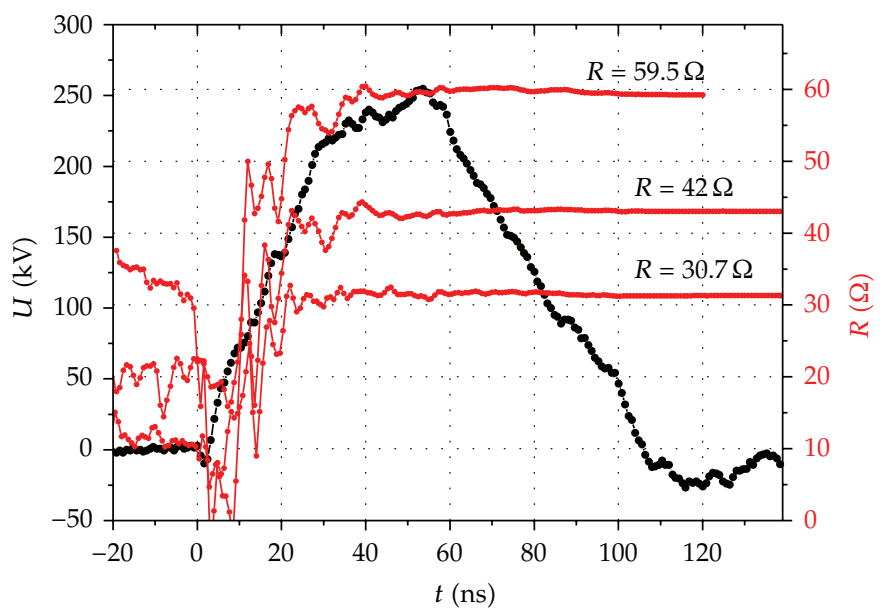

Figure 2: TEU-500 accelerator calibration using various load resistors.

Figure 4 shows typical waveforms of voltage and total electron beam current for a diode with a graphite cathode of diameter $45 \mathrm{~mm}$, A-K gap $15 \mathrm{~mm}$.

Curves are averaged for 10 pulses at a frequency of 1 pulse/s after running the cathode for 10-20 pulses. In this range of experiments a metal grid was used as an anode, while the current recorded by the Faraday cup was adjusted for the value of grid optical transparency [20]. Figure 5 shows the experimental values of the perveance of the diode during electron beam generation and calculated values obtained from ratio (1.2) when $v=2 \mathrm{~cm} / \mu \mathrm{s}$.

Results show that for the graphite cathode with a diameter of $45 \mathrm{~mm}, 20 \mathrm{~ns}$ after voltage to the diode is applied the current-voltage characteristics of the diode are satisfactorily described by the Child-Langmuir law (except for the initial 10-20 ns). The contribution of the periphery of the cathode to total electron current appears only in the increase in surface area of the emission, caused by the expansion of the explosive emission surface across the A-K gap. When widening the A-K gap from 10 to $17 \mathrm{~mm}$, diode impedance increases from 30 to $100 \mathrm{Ohms}$. The use of an autotransformer in the TEU-500 electron accelerator enables a significant extension in the range of the diode impedance, in which there is an alignment of the diode with the generator. 


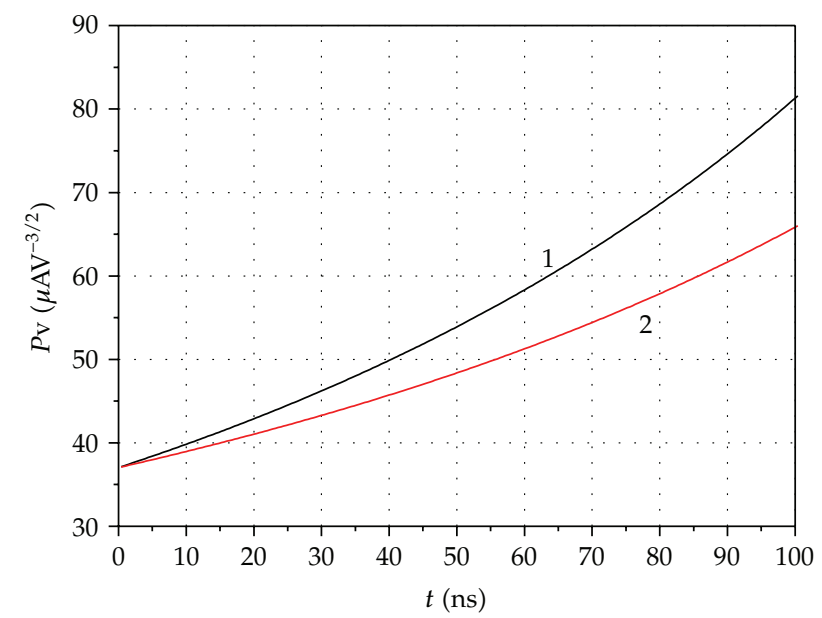

Figure 3: Calculated time-dependent change in perveance of the planar diode, taking into account (1) both the reduction of the A-K gap and increase in cathode area (radial plasma expansion); (2) only the reduction of the A-K gap.

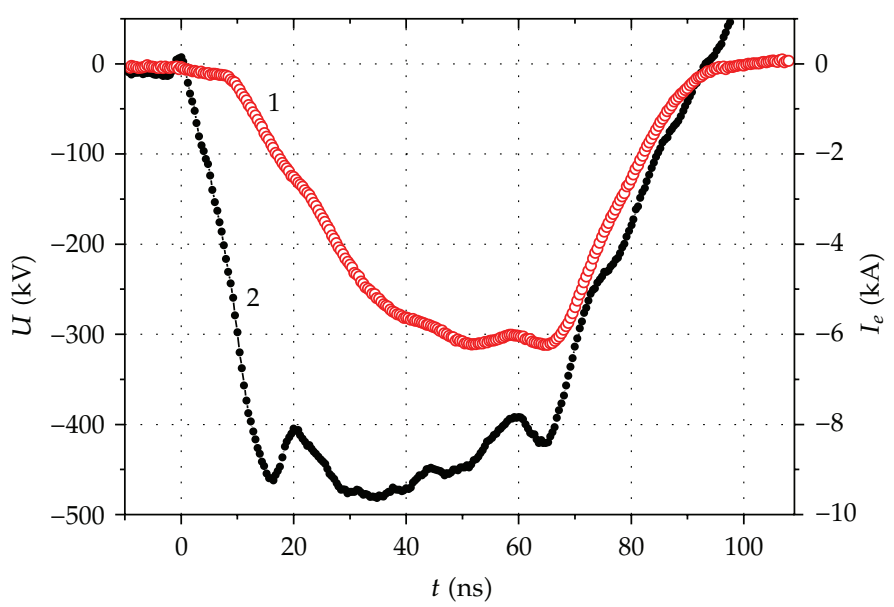

Figure 4: Waveforms of (1) electron current and (2) voltage.

In Figure 6 the energy density distribution over a beam cross-section, measured by both a radiochromic film [21] and an infrared camera, is shown. The target made of foam has been pre-cut in half across the diameter to record the temperature field distribution inside the target. Afterwards it was opened and a thermal field of the electron beam at the inner surface was viewed by an infrared camera. The temperature values were further recalculated into the energy density of the electron beam.

It is shown that the energy density distribution of the electron beam over cross-section is homogeneous (there is no increase in the current density on the cathode periphery), but a diameter of the thermal imprint formed by the beam exceeds the diameter of the cathode. In [20] it is shown that the electron beam divergence in the A-K gap caused by a distortion in the electric field at the periphery of the cathode is the main source of parasitic losses in planar diodes. The half-angle of divergence is $68^{\circ}$ at small A-K gaps and decreases to $60^{\circ}$ 


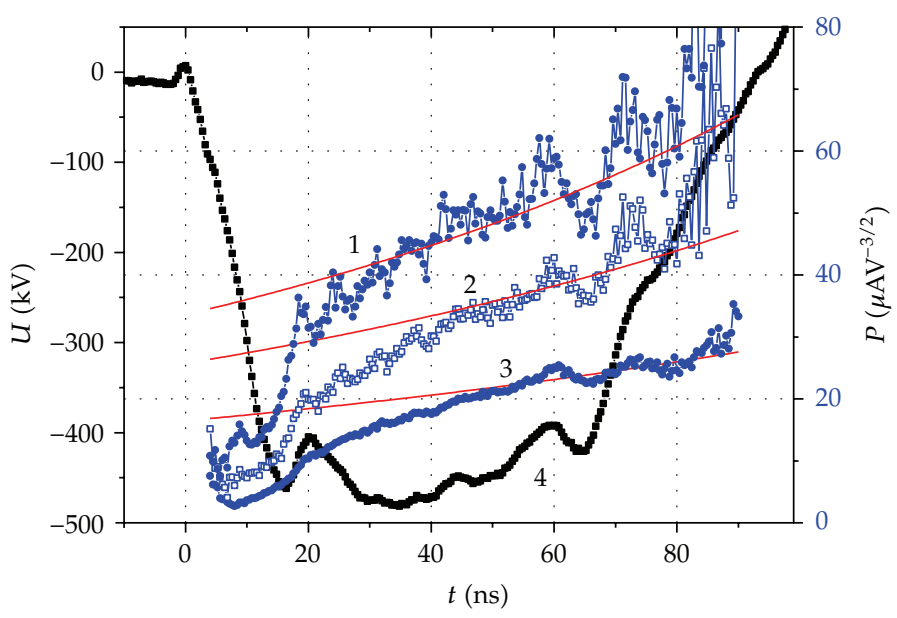

Figure 5: Experimental (dots) and calculated (solid line) values of the perveance of the planar diode with a graphite cathode with a diameter of $45 \mathrm{~mm}$ and gap of (1) 11, (2) 12.5, and (3) $14 \mathrm{~mm}$. (4) represents a waveform of voltage.

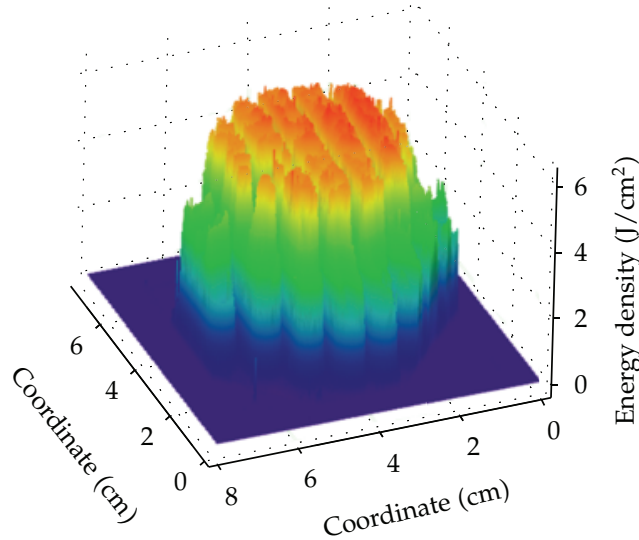

(a)

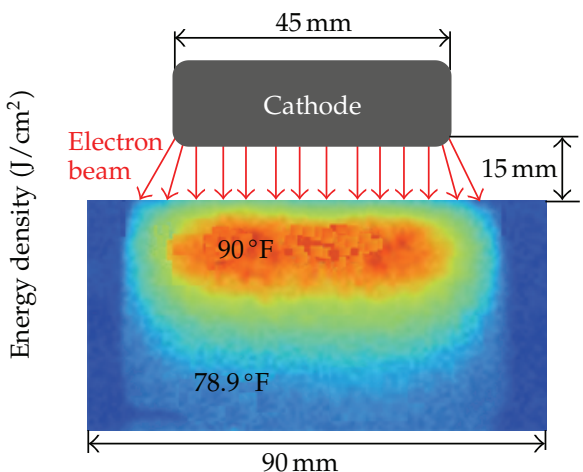

(b)

Figure 6: Distribution of the energy density over the cross-section of electron beam, measured by a radiochromic film, (a) and temperature distribution along the depth of a foam sample, measured by infrared camera (b).

with an increase in the gap. It is important to note that an increase in the diameter of the electron beam does not cause an increase in electron current compared with the calculated value obtained from ratio (1.1). The main part of the electron space charge is concentrated at the cathode region of less than $0.1-0.3 \mathrm{~mm}$ thickness. This charge limits the current density.

In 2008-2009, we carried out studies on the voltage-current characteristics of a planar diode during pulse electron beam generation with a current density of $0.2-0.4 \mathrm{kA} / \mathrm{cm}^{2}$, electron energy of $350-450 \mathrm{keV}$, and pulse power of $1-3 \mathrm{GW}$, with diode impedance in agreement with output resistance of the generator of nanosecond pulses [22]. The results of experimental investigations of a diode with explosive emission cathode made of graphite, carbon fabric, and copper (solid and multiple point) were analysed together with a 
multipoint tungsten cathode. It was found that the electron current of the diode was satisfactorily described by the Child-Langmuir law, provided the reduction of the A-K gap and increase in emission surface area due to the expanding plasma are taken into account.

\section{Investigation of a Planar Diode with a Cathode Diameter of $60 \mathrm{~mm}$}

If the diameter of a flat explosive emission cathode is more than $50 \mathrm{~mm}$, an increase in emission surface area due to the radial plasma expansion does not exceed $10 \%$ within the first 100 ns. Therefore, the contribution of edges to diode operation does not affect the accuracy of accelerating voltage and electron current measurements. The operation of a diode with an explosive emission cathode can be controlled according to how an emission surface area changes during electron beam generation. The area of the emission surface of the cathode can be calculated from the ratio of experimental current and calculated values of electron current density, the latter being obtained from ratio (1.1). The dependences obtained are shown in Figure 7.

For graphite cathodes with different areas, in the early stage of electron beam generation the process of emitting area increase follows the same general pattern. However for the cathode with a smaller diameter, during the voltage pulse, explosive emission plasma has time to cover its entire surface, while, for the cathode with an area of $28 \mathrm{~cm}^{2}$, for $80 \mathrm{~ns}$ an emitting plasma surface is formed on only $80-85 \%$.

The results show that, with an increase in the A-K gap, the rate of plasma formation on the cathode surface varies slightly. Figure 8 shows the effect of a change in the area of the emitting surface of a graphite cathode with a diameter of $60 \mathrm{~mm}$, during the generation of a pulse of electron current at the A-K gap of 10.5, 12, and $15 \mathrm{~mm}$. Figure 8 also shows a change in the $60 \mathrm{~mm}$ diameter cathode emitting surface area, made of carbon fabric. The presence of micro-inhomogeneities on the cathode surface greatly facilitates and accelerates the process of emission surface formation.

The coincidence of experimental and calculated values of the cathode emitting surface area demonstrates that, when the A-K gap is increased from 10 to $15 \mathrm{~mm}$, the electron current of a planar diode with a graphite cathode is limited only by space charge in the gap. The contribution of the periphery of the cathode to total electron current during the first 80-100 ns does not exceed measurement error.

\section{Investigation of a Diode with an Explosive Emission Cathode during 300-600 ns}

The TEU-500 electron accelerator only allows for an 80-100 ns study of the edge contribution in a diode with an explosive emission cathode. The conditions for the development and formation of explosive emission plasma during the first stage of pulsed ion beam generation (negative polarity pulse) are analogous to conditions in an electron diode with an explosive emission cathode [23]. Investigations were conducted on a TEMP-4M ion accelerator with the following parameters: a negative first pulse $(300-600 \mathrm{~ns}, 150-200 \mathrm{kV})$ followed by a positive second pulse $(150 \mathrm{~ns}, 250-300 \mathrm{kV})$; pulse frequency 5-10 pulses per minute. The diode connection and the diagnostic equipment used are described in more detail in our papers $[23,24]$. Figure 9 displays a waveform of the voltage on the potential electrode and electron current. These results were obtained using a $5.2 \mathrm{Ohm}$ resistor during calibration. Calculation of the voltage was performed using the relation $U_{\text {calc }}(t)=R_{\text {load }} \cdot I(t)+L_{\text {load }} \cdot(d I / d t)$, where 


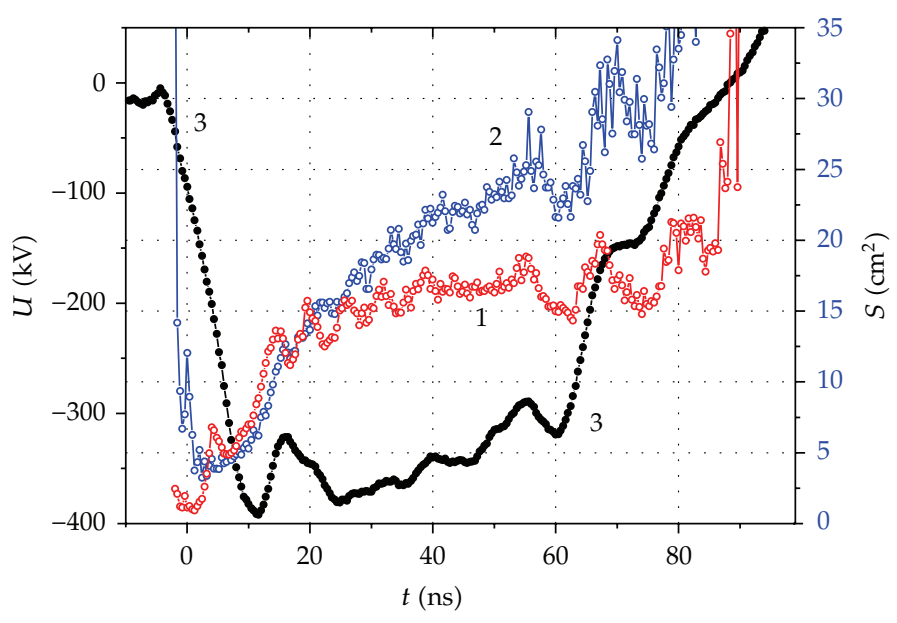

Figure 7: Change in cathode emitting surface during electron current generation, for cathodes of diameter (1) $45 \mathrm{~mm}, S=16 \mathrm{~cm}^{2}$ and (2) $60 \mathrm{~mm}, S=28 \mathrm{~cm}^{2}$. (3) represents a waveform of voltage.

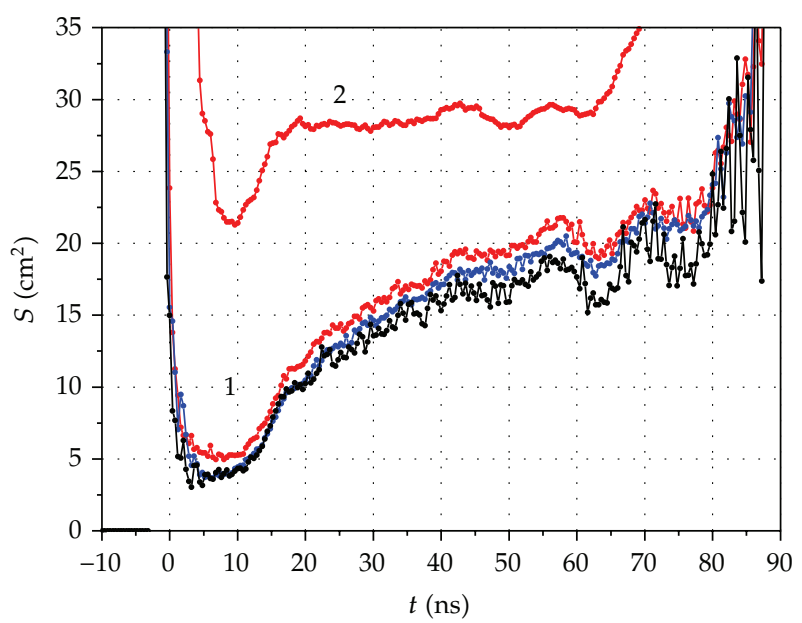

Figure 8: Change in cathode emitting surface during the generation of a pulse of current for a $60 \mathrm{~mm}$ diameter cathode made of (1) graphite and (2) carbon fabric.

$L_{\text {load }}$ is parasitic load inductance $240 \mathrm{nH}$. Calculation of the inductance was made from the ratio for a straight conductor at a frequency of 1-10 $\mathrm{MHz}$ [25]. We investigated a planar diode $(4.5 \mathrm{~cm} \times 22 \mathrm{~cm})$ with a graphite potential electrode (the cathode during the first negative pulse and the anode on the second positive pulse). A gap of 7.5-9 $\mathrm{mm}$ between the potential and grounded electrodes was chosen so that diode impedance matched the wave impedance of the Blumlein.

In space charge limitation mode, the current density of the singly ionized carbon ion $\mathrm{C}^{+}$is $0.7 \%$ of electron current density. Optimization of the design and operating modes of the ion diode with magnetic self-insulation resulted in increased efficiency of ion current generation by a factor of 10-12 times [23]. At the same time, the ionic component of current did not exceed $10 \%$ of total diode current. Therefore, remaining within the measurement accuracy of the voltage-current characteristics, the dynamics of the explosive emission 


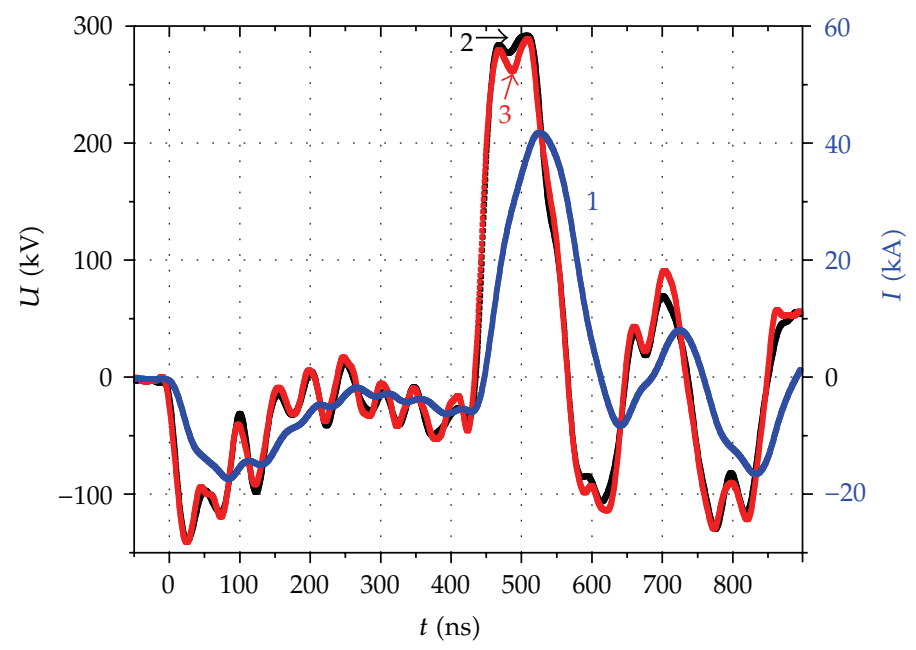

Figure 9: Waveforms of (1) current, (2) experimental, and (3) calculated voltage.

plasma in the A-K gap can be controlled by the Child-Langmuir law for electron current component. Diode impedance is then

$$
R_{\mathrm{calc}}(t)=\frac{U}{S \cdot J_{e}}=\frac{\left(d_{0}-v t\right)^{2}}{2.33 \cdot 10^{-6} S \cdot U^{1 / 2}}
$$

where $S$ is the area of the potential electrode of the diode.

Figure 10 displays experimental and calculated values of diode impedance.

Once the potential electrode surface is covered with plasma, total diode current is limited only by the electron space charge in the A-K gap (300 ns $<t<600 \mathrm{~ns}$ in Figure 10). Experimental values of diode impedance are well described by (5.1), in which plasma expansion speed is constant and equal to $1.3 \pm 0.2 \mathrm{~cm} / \mu \mathrm{s}$. When calculating diode impedance, an amplification coefficient of the electron current was taken to be equal to 1. Within $600 \mathrm{~ns}$ after voltage to the diode is applied, the influence of the anode plasma on diode impedance was insignificant.

\section{Investigation of the Ion Beam Generation}

In the space charge limitation mode ion current density is defined by the Child-Langmuir law also. In this case when calculating the ion current it is necessary to consider not only the reduction in the A-K gap due to plasma heat expansion as in relation (1.1) but also the increasing of the effective gap spacing due to the plasma erosion [26] when the polarity on the potential electrode changes. The ion current is equal to

$$
I_{\text {ion }}(t)=\frac{4 \varepsilon_{0} \sqrt{2 z}}{9 \sqrt{m_{i}}} \cdot \frac{U^{3 / 2} \cdot S}{\left[d_{0}-v \cdot\left(t-t_{0}\right)\right]^{2}},
$$

where $m_{i}$ and $z$ are the ion mass and charge, respectively, and $t_{0}$ is the time of arrival of the second pulse $\left(t_{0}=430 \mathrm{~ns}\right.$ in Figure 9), $t$ is the current time of voltage. 


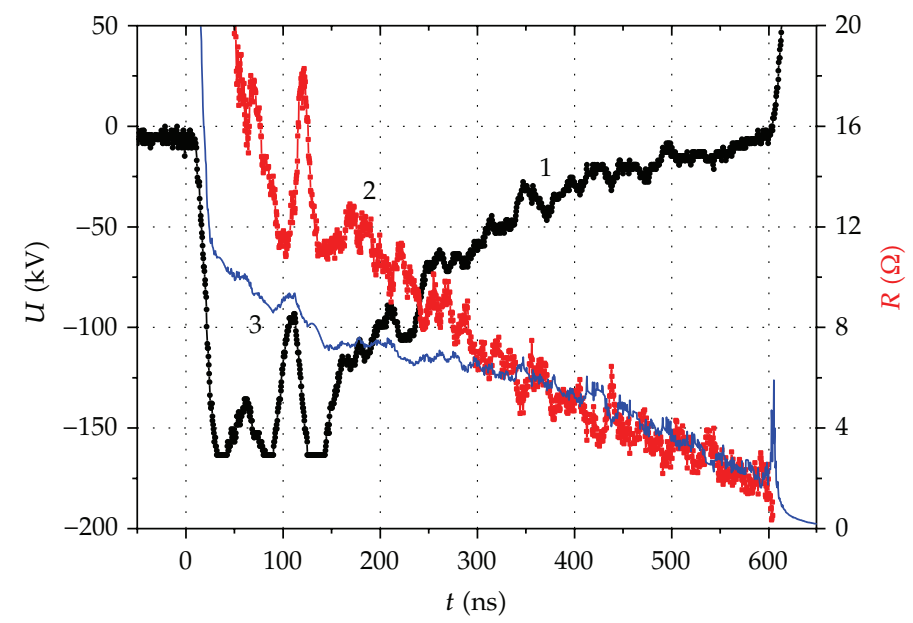

Figure 10: Waveforms of (1) voltage, (2) experimental, and (3) calculated impedance values.

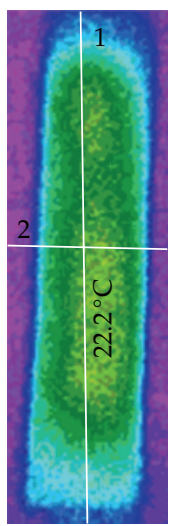

(a)

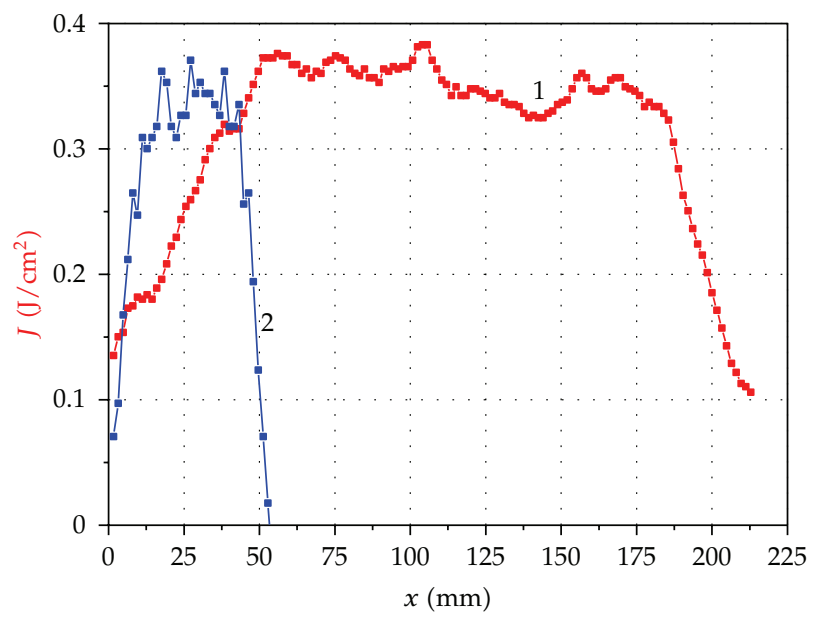

(b)

Figure 11: Thermal imprint (a) and ion beam energy density distribution at the target (b) in the vertical section (1) and horizontal section (2).

Figure 11 shows the energy density distribution over the cross-section of the ion beam formed by the TEMP-4M accelerator. The distribution of energy density was measured by means of a thermal imaging technique [27]. The energy density of the ion beam was calculated by the temperature destitution on a brass target intercepting the beam. The target was located at a distance of $4 \mathrm{~cm}$ from the diode.

It is evident that ion beam is uniform on the entire potential electrode surface. Influence of the periphery of the potential electrode is not significant.

\section{Conclusion}

This paper has presented experimental studies of a planar diode with an explosive emission cathode, with diode impedance in agreement with the output resistance of the generator 
of nanosecond pulses. Diode operation during the generation of an electron beam can be divided into two stages. In the first stage, immediately after the voltage is applied to the diode, diode current is limited by emissivity of the cathode. For a graphite cathode with a diameter of $45 \mathrm{~mm}$, the duration of the first stage is 15-20 ns, increasing to 30-40 ns at lower voltage amplitude. Diode perveance during the first stage of diode operation is much less than calculated perveance. An increase in cathode area to $28 \mathrm{~cm}^{2}$ increases the duration of the formation of a homogeneous emission surface on the cathode, and, by the end of the voltage pulse, electrons are emitted only from $80 \%$ of the cathode surface area. An increase in the A-K gap slightly alters the rate of continuous emission surface expansion on the cathode.

In the second stage, electron current is limited by space charge in the A-K gap, with current-voltage characteristics of the diode being well described by the Child-Langmuir law only when a reduction of the A-K gap and an increase in the area of the emission surface, caused by plasma expanding at a constant velocity, are taken into account.

The contribution of the cathode periphery to total electron current results from an increase in the emissive surface area caused by the expansion of explosive emission plasma across the A-K gap. If the area of a flat cathode is more than $25 \mathrm{~cm}^{2}$, the edge contribution does not exceed the measurement error of the total electron current of the diode.

\section{Acknowledgment}

This work was supported by the Russian target program "The Scientific brainpower of Innovative Russia" 2009-2013, ГК no. 14.740.11.1181.

\section{References}

[1] S. P. Bugaev, Y. I. Krenydel, and P. M. Shanin, Electron Beams of a Wide Profile, M Energoatomizdat, 1984.

[2] G. A. Mesyats, Pulse Energetics and Electronics, Science, Moscow, Russia, 2004.

[3] G. A. Mesyats and D. I. Proskurovski, Pulsed Electric Discharge in a Vacuum, Science, Novosibirsk, Russia, 1984.

[4] D. Yarmolich, V. Vekselman, V. T. Gurovich, J. Z. Gleizer, J. Felsteiner, and Y. E. Krasik, “Micron-scale width multislot plasma cathode," Physics of Plasmas, vol. 15, no. 12, Article ID 123507, 2008.

[5] I. Langmuir, "The effect of space charge and residual gases on thermionic currents in high vacuum," Physical Review, vol. 2, no. 6, pp. 450-486, 1913.

[6] A. F. Shubin and Ya. Ya. Yurike, "The growth in current in the initial stage of vacuum breakdown between planar electrodes at a slowly increasing voltage," Proceedings of Universities, Physics, vol. 157, no. 6, p. $134,1975$.

[7] R. K. Parker, R. E. Anderson, and C. V. Duncan, "Plasma-induced field emission and the characteristics of high-current relativistic electron flow," Journal of Applied Physics, vol. 45, no. 6, pp. 2463-2479, 1974.

[8] S. Y. Belomyttsev, S. D. Korovin, and I. V. Pegel', "Current in a high-current planar diode with a discrete emitting surface," Technical Physics, vol. 44, no. 6, pp. 695-699, 1999.

[9] G. Djogo and J. D. Cross, "Circuit modeling of a vacuum gap during breakdown," IEEE Transactions on Plasma Science, vol. 25, no. 4, pp. 617-624, 1997.

[10] A. Roy, R. Menon, S. Mitra et al., "Impedance collapse and beam generation in a high power planar diode," Journal of Applied Physics, vol. 104, no. 1, Article ID 014904, 2008.

[11] A. Roy, S. Mitra, R. Menon et al., "Prepulse suppression on a high-power electron beam accelerator using a dielectric cathode holder," IEEE Transactions on Plasma Science, vol. 37, no. 1, pp. 67-75, 2009.

[12] L. Li, L. Liu, H. Wan, J. Zhang, J. Wen, and Y. Liu, "Plasma-induced evolution behavior of spacecharge-limited current for multiple-needle cathodes," Plasma Sources Science and Technology, vol. 18, Article ID 015011, 2009.

[13] A. Roy, R. Menon, S. K. Singh et al., "Shot to shot variation in perveance of the explosive emission electron beam diode," Physics of Plasmas, vol. 16, no. 3, Article ID 033113, 2009. 
[14] F. Hegeler, M. Friedman, M. C. Myers, J. D. Sethian, and S. B. Swanekamp, "Reduction of edge emission in electron beam diodes," Physics of Plasmas, vol. 9, no. 10, p. 4309, 2002.

[15] A. Roy, R. Menon, S. Mitra et al., "Intense relativistic electron beam generation and prepulse effect in high power cylindrical diode," Journal of Applied Physics, vol. 103, no. 1, Article ID 014905, 2008.

[16] G. Remnev, A. Pushkarev, and V. Ezhov, "Investigation of planar explosive emission diode parameters during the pulsed electron beam generation," in Proceedings of the 5th International Symposium on Pulsed Power and Plasma Applications, pp. 161-167, Korea, 2004.

[17] G. Remnev, E. Furman, A. Pushkarev, N. Kondratiev, and D. Goncharov, "High-current pulsed accelerator with matching transformer : construction and exploitation characteristics," IEEJ Transactions on Fundamentals and Materials, vol. 124, no. 6, p. 491, 2004.

[18] G. E. Remnev, A. I. Pushkarev, and E. G. Furman, "Matching a double forming line to explosiveemission diode," Technical Physics Letters, vol. 30, no. 7, pp. 603-604, 2004.

[19] Y. I. Isakova, A. I. Pushkarev, and G. E. Kholodnaya, "A differential high-voltage divider," Instruments and Experimental Techniques, vol. 54, no. 2, pp. 183-186, 2011.

[20] A. I. Pushkarev, Y. N. Novoselov, and R. V. Sazonov, "Losses in a pulsed electron beam during its formation and extraction from the diode chamber of an accelerator," Instruments and Experimental Techniques, vol. 50, no. 5, pp. 687-694, 2007.

[21] G. Remnev, A. Pushkarev, and V. Ezhov, "The study on the energy density of high-current pulsed electron beam," Bulletin of the Tomsk Polytechnic University, vol. 308, no. 6, p. 76, 2005.

[22] A. I. Pushkarev and R. V. Sazonov, "Research of cathode plasma speed in planar diode with explosive emission cathode," IEEE Transactions on Plasma Science, vol. 37, no. 10, p. 1901, 2009.

[23] A. I. Pushkarev, J. I. Isakova, M. S. Saltimakov, and R. V. Sazonov, "Investigation of magnetically selfinsulated effect in an ion diode with an explosive emission potential electrode," Physics of Plasmas, vol. 17, no. 1, Article ID 013104, 2010.

[24] A. I. Pushkarev, J. I. Isakova, M. S. Saltimakov, and R. V. Sazonov, "Research on the plasma dynamics in a magnetically self-insulated ion diode with explosive emission potential electrode," Natural Science, vol. 2, no. 5, pp. 419-426, 2010.

[25] P. L. Kalantarov and L. A. Tseydlin, Calculation of the inductances. Reference-Book, Energoatomizdat, Leningrad, Russia, 1986.

[26] S. Humphries, Charged Particle Beams, John Wiley \& Sons, New York, NY, USA, 1990.

[27] H. A. Davis, R. R. Bartsch, J. C. Olson, D. J. Rej, and W. J. Waganaar, "Intense ion beam optimization and characterization with infrared imaging," Journal of Applied Physics, vol. 82, no. 7, pp. 3223-3231, 1997. 

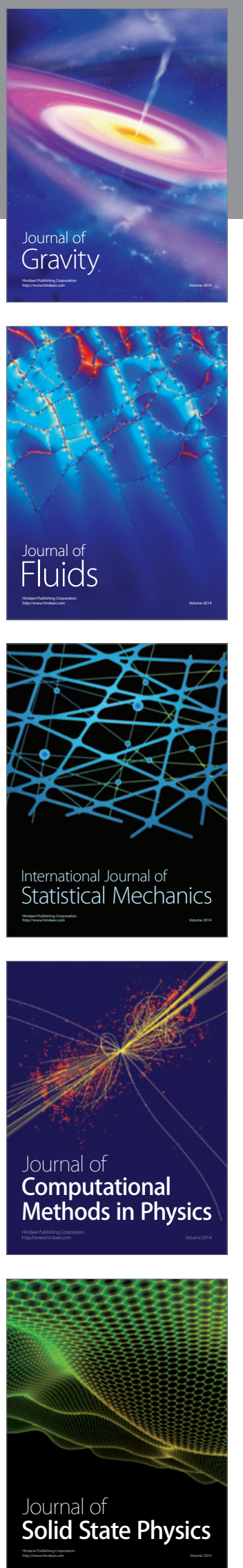

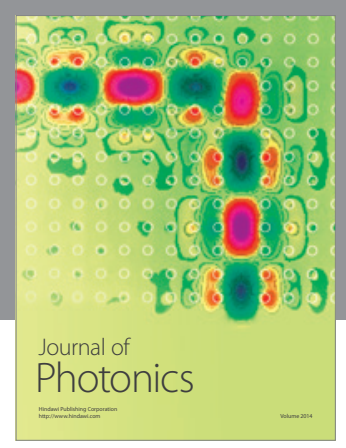

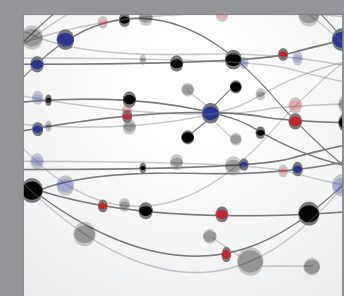

The Scientific World Journal
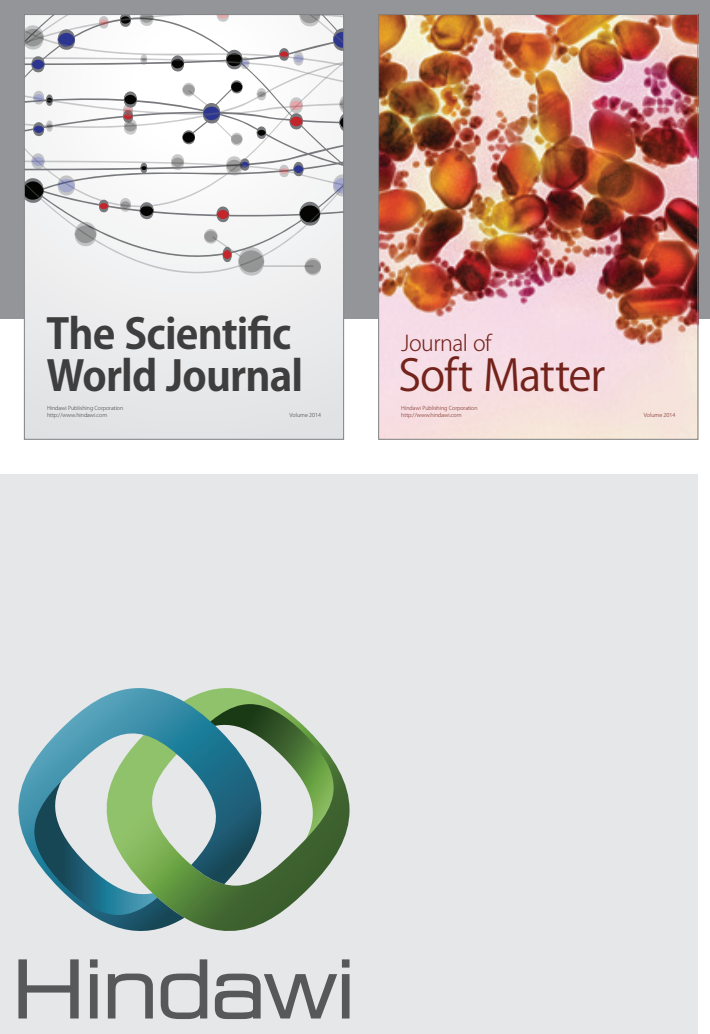

Submit your manuscripts at

http://www.hindawi.com
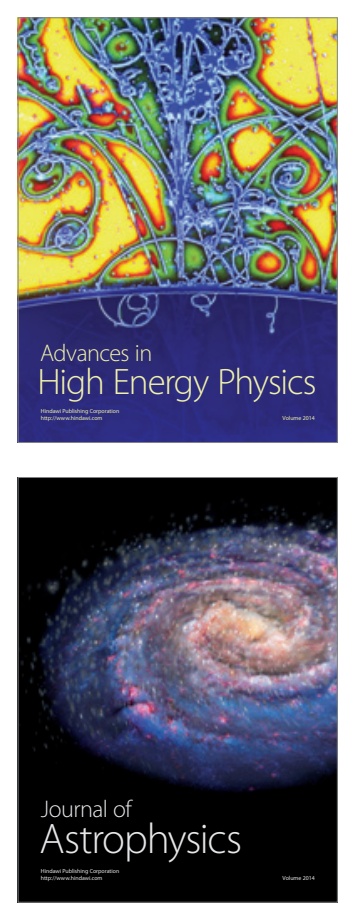
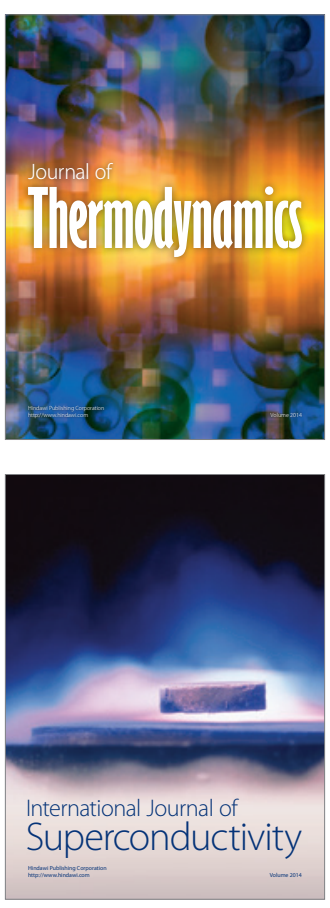
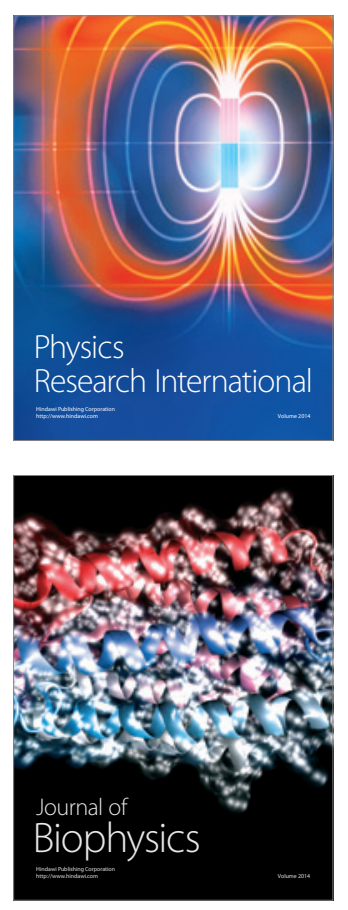
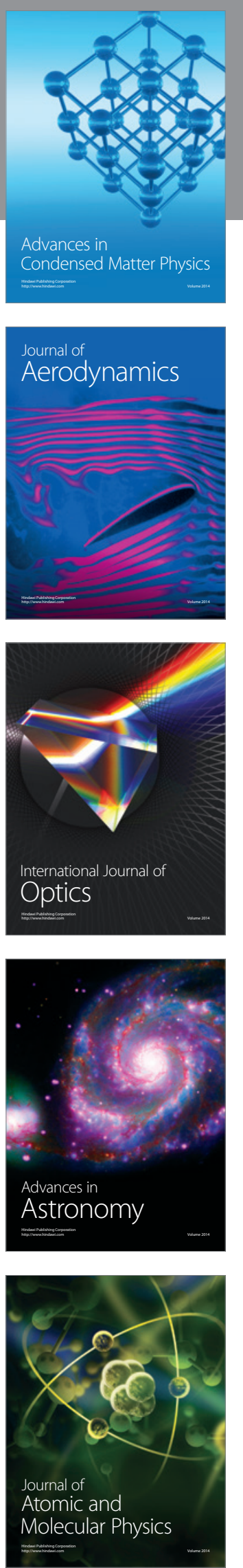